\title{
The importance of being transparent
}

\author{
In recent years, questions have been raised regarding the failure of some \\ preclinical work to translate to clinical benefit and the inability to reproduce \\ some high-profile studies. While myriad factors contribute to these \\ problems, an important step in improving the integrity of published work is \\ for journals to enforce rigorous reporting of methods and results.
}

In November 2014, the JCI revised its instructions to authors for manuscripts and our data reporting requirements (http://www.jci.org/kiosks/authors). These changes stem from an ongoing series of efforts by the journal to improve upon our reporting of data (1-3) and also in part from a larger conversation that's taking place in the biomedical publishing community about transparency and reproducibility (4). The Editorial Board of the JCI is committed to ensuring that all our articles adhere to the highest standard of data rigor and transparency.

Our latest requirements focus on sample size and statistical analysis. While it may seem like common sense, all too often it has been difficult to locate the exact number of samples or animals in preclinical studies and the statistical test used to assess differences. We now require both to be reported in the figure legends for all manuscripts. Additionally, we are asking authors to indicate the number of experiments that were conducted for any representative experiment. We continue to recommend that authors avoid the use of bar graphs, instead plotting each individual data point to allow readers to better appreciate the sample size and distribution (3).

Our biostatistician colleagues often lament that many preclinical research studies are woefully underpowered in terms of sample size and that the research community is often prone to overinterpreting the meaning of $P$ values (5). We at the JCI believe that clearly showing the data points and related statistical analysis is the best way for readers to discern the strength of the data and any conclusions made. We've also noted some common errors being made in terms of the choice of statistical analysis utilized - most prominently a failure to take into account multiple comparisons and repeated measures and to ensure that the data meet the distribution assumptions of the test. The statistical analysis of data will only be meaningful if the appropriate test is applied. As a community, we often need to be savvier about understanding statistics, and it may be necessary to seek out the appropriate experts to help design and analyze studies. At the JCI, we are fortunate to have a panel of biostatisticians, Barry Moser, Maren Olsen, and Cynthia Coffman, whom the editors can call upon for more detailed scrutiny of statistical methods in manuscripts.

In addition to requiring more complete description of statistical methods, we are also asking authors to provide access to highthroughput data sets. We have previously required authors to deposit gene-expression microarray data in a MIAME-compliant database; we've now included a requirement to deposit other types of arrays and highthroughput sequencing data in a MINSEQEcompliant database (such as GEO, EMBLEBI, or DDBJ). We encourage the deposition of other large data sets whenever possible.

The JCI is also now requiring additional information in the Methods section, such as minimum reporting standards for newly synthesized chemical compounds, including the structure, synthesis, and purity of the compound. Further, we're asking authors to include more information about antibodies utilized and the source of cell lines in their studies.

Lastly, the JCI continues to require authors to include uncropped and unedited versions of all immunoblots and gels for review by the editors and reviewers. We encourage authors to include these images with their publication on our website as well. Although most manuscripts do not have issues, it is not uncommon for us to see figures that lack appropriate indication of splice sites and figure panels that present blots derived from different gels. We manually screen every blot to be published in the journal to ensure the highest data integrity, requiring authors to clearly indicate splicing and instances in which parallel samples were run on different gels. Here again, we believe our readers can better interpret that data when the results are clearly presented.

We believe that reporting of all information related to materials, samples, and statistics is of paramount importance for researchers who need to accurately assess the data and conduct follow-up experiments in their own labs. The JCI has never had a restriction on the length of the Methods section, and we allow additional Methods to be included in the supplemental materials.

All of these efforts are aimed at improved reporting of data from authors, which in turn eases interpretation of data by the wider community. We are all aware that there have been numerous high-profile retractions in recent years as well as a number of prominent studies documenting irreproducibility $(6,7)$. While we recognize that these requirements cannot solve all of the problems that led to these different issues, we feel that this is an important step to ensure that authors present the highest quality and integrity of data in the JCI.

\section{Sarah Jackson, Senior Science Editor}

1. Neill US, Turka, LA. Navigating through the gray (and CMYK) areas of figure manipulation: rules at the JCI. JClin Invest. 2007;117(10):2736-2736.

2. Neill US. All data are not created equal. J Clin Invest. 2009;119(3):424.

3. Rockman HA. Great expectations. JClin Invest. 2012;122(4):1133.

4. [No authors listed]. Journals unite for reproducibility. Nature. 2014;515(7525):7.

5. Nuzzo R. Scientific method: statistical errors. Nature. 2014;506(7487):150-152

6. Normile D, Vogel G. Stem cells. Irreproducibility dogs new reprogramming method. Science. 2014;343(6177):1299-1300.

7. Begley CG, Ellis LM. Drug development: Raise standards for preclinical cancer research. Nature. 2012;483(7391):531-533. 